TITLE:

\title{
Impact of Directional Antenna of Primary System Receiver in Spectrum Sensing: A Case Study
}

\section{$\operatorname{AUTHOR(S):~}$}

Yagi, Yoshimitsu; Ohno, Takuto; Murata, Hidekazu; Yamamoto, Koji; Yoshida, Susumu

\section{CITATION:}

Yagi, Yoshimitsu ... [et al]. Impact of Directional Antenna of Primary System Receiver in Spectrum Sensing: A Case Study. IEEE Communications Letters 2011, 15(3): 308-310

\section{ISSUE DATE:}

2011-03

URL:

http://hdl.handle.net/2433/139537

\section{RIGHT:}

(c) 2011 IEEE. Personal use of this material is permitted. Permission from IEEE must be obtained for all other uses, in any current or future media, including reprinting/republishing this material for advertising or promotional purposes,

creating new collective works, for resale or redistribution to servers or lists, or reuse of any copyrighted component of this work in other works.; This is not the published version. Please cite only the published version.; この論文は出版社版

でありません。引用の際には出版社版をご確認ご利用ください。 


\title{
Impact of Directional Antenna of Primary System Receiver in Spectrum Sensing: A Case Study
}

\author{
Yoshimitsu Yagi, Takuto Ohno, Hidekazu Murata, Member, IEEE, Koji Yamamoto, Member, IEEE, \\ and Susumu Yoshida, Member, IEEE,
}

\begin{abstract}
In cognitive radios, a directional antenna can be used at the primary system receiver in order to improve reception performance. The problem is that the cognitive radio sensors may not detect weak signals from the primary system transmitter far away, whereas these signals can be received by a highsensitivity primary system receiver. The present paper describes the experimental results obtained in field trials performed in order to demonstrate the impact of various parameters (e.g., directional gain, reflections, and diffractions) in spectrum sensing.
\end{abstract}

Index Terms - cognitive radio, experimental study, spectrum sensing, directional antenna.

\section{INTRODUCTION}

$\mathbf{I}$ $\mathrm{N}$ cognitive radio systems [1], [2], a directional antenna can be used at the primary system receiver to receive weak signal from the transmitter far away. The directional antenna provides the advantages of higher gain because, unlike the omnidirectional antenna, the directional antenna can focus energy in one particular direction. Furthermore, the directional antenna at the fixed primary system receiver is generally mounted on the roof of the building in order to establish LOS.

On the other hand, the mobile cognitive radio often uses an omnidirectional antenna. The antenna height is much lower than the building height because the cognitive radio is a mobile station. In order to overcome these disadvantages, the cognitive radio must have better sensitivity than the primary system. However, it is unrealistic to drastically reduce the noise figure or take the long averaging time. The problem arises when the cognitive radio must robustly detect a weak signal from the primary system transmitter. The cognitive radio may not detect weak signals from the transmitter in the primary system, whereas these signals can be received by the high-sensitivity receiver in the primary system (e.g., using the directional antenna). As a result, the cognitive radio may not protect the communication of the primary system.

The required sensitivity for the cognitive radio is dependent on the sensitivity of the primary system and the relative positions of the cognitive radio and primary system. Therefore, we must take into account 3D patterns for the directional antenna, reflections, and diffractions outside the building. The present paper describes experimental results obtained through field trials conducted as a case study. Through this case study, we

Y. Yagi, T. Ohno. H. Murata, K. Yamamoto, and S. Yoshida are with the Graduate School of Informatics, Kyoto University, Kyoto, Japan (e-mail: contact-h22@hanase.kuee.kyoto-u.ac.jp).

Manuscript received August 12, 2010; revised August 12, 2010.

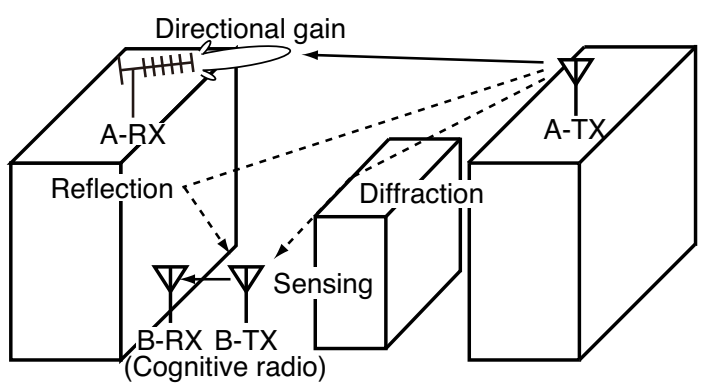

Fig. 1. System scenario. Group A is a high-priority system, and group $\mathrm{B}$ is a cognitive radio system (low-priority system). A-RX uses a directional antenna. We must take into account the 3D pattern for the directional antenna, reflections, and diffractions outside the building.

demonstrate the impact of various parameters (e.g., directional gain, reflections, and diffractions) in spectrum sensing.

\section{SYSTEM SCENARIO}

As shown in Fig. 1, two source-destination pairs, group A and group $\mathrm{B}$, share the same frequency band. The stations in group A are high-priority (primary) users, whereas the stations in group B are cognitive radios, which are considered to be low-priority (secondary) users. A-RX uses a directional antenna and receives a weak signal from A-TX. Group A does not provide any information for spectrum sharing (e.g., the signal level from B-TX received at A-RX) to group B. Moreover, group $\mathrm{B}$ has no position information pertaining to group A.

In order to avoid interference with A-RX, B-TX does not start transmission to B-RX when the presence of ATX is detected. For simplicity, we use the energy detection method described in [3], [4] instead of cyclostationary feature detection [5]. In this experiment, we ensure that only BTX detects the presence of A-TX. Here, the decision of the presence of A-TX is defined by $D$ and is given by

$$
D= \begin{cases}0 & P_{\mathrm{A}, \mathrm{B}-\mathrm{TX}}<T_{\text {detect }}, \\ 1 & P_{\mathrm{A}, \mathrm{B}-\mathrm{TX}} \geq T_{\text {detect }},\end{cases}
$$

where $P_{\mathrm{A}, \mathrm{B}-\mathrm{TX}}$ is the signal level received by B-TX from ATX, and $T_{\text {detect }}$ is the signal sense threshold. In other words, if $P_{\mathrm{A}, \mathrm{B}-\mathrm{TX}}$ does not exceed the signal detection threshold $T_{\text {detect }}$, B-TX starts transmission to B-RX.

\section{HARDWARE ARCHITECTURE}

Fig. 2 shows the hardware architecture of B-TX and B-RX. $\mathrm{B}-\mathrm{TX}$ is composed of a sensing part and a communication 


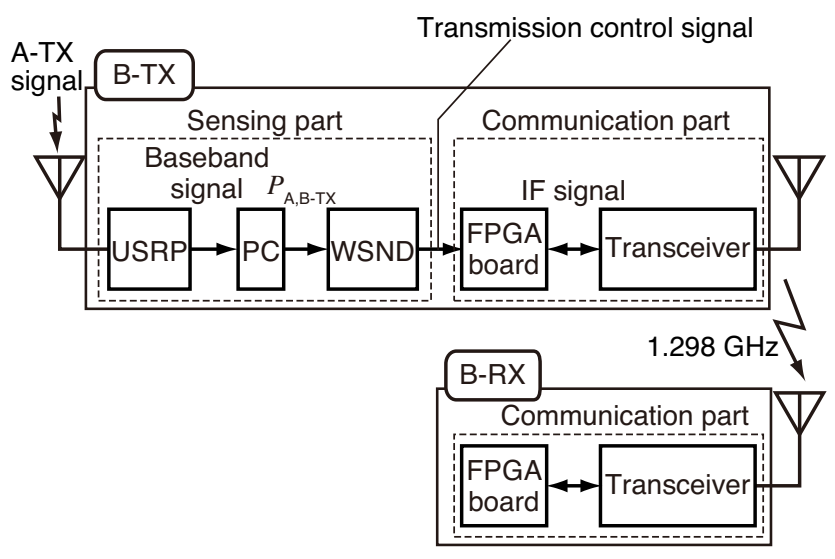

Fig. 2. Hardware architecture of B-TX and B-RX. A-TX and A-RX have the same architecture as B-RX. "WSND" denotes a wireless sensor network device.

part. The communication part includes a transceiver and an FPGA board for data communication over the $1.2-\mathrm{GHz}$ band. The sensing part includes a wireless sensor network device (referred to hereinafter as WSND), a universal software radio peripheral (USRP) [6], and a personal computer (PC) for sensing A-TX. The stations in group A have only the communication part, similar to B-RX.

\section{A. Sensing part}

1) WSND: This device is equipped with a 2.4-GHz IEEE 802.15.4 radio chip, general-purpose I/O pins, a battery, a $\mathrm{CPU}$, and other components. In addition, this device can be programmed using Java. In this experiment, the WSND is used to determine whether signal transmission should be carried out. If $P_{\mathrm{A}, \mathrm{B}-\mathrm{TX}}$ does not exceed $T_{\text {detect }}$, the WSND of B-TX outputs the signal via a general-purpose I/O pin in order to allow transmission from the communication part.

2) USRP: The USRP system consists of a motherboard and changeable plug-in daughterboards. The motherboard and daughterboard perform interpolation/decimation, up/downconversion, signal amplification, and conversion to/from the analog and digital signal domains. The host $\mathrm{PC}$ receives $\mathrm{I}$ and $\mathrm{Q}$ signals through the USB. The USRP complements the GNU Radio software for the development of software radios. GNU Radio is an open-source software toolkit that provides a library of signal processing blocks for developing communication systems.

In this experiment, the USRP is used as an energy detector. In this method, the processing gain is proportional to the FFT size $N$ and the number of observation/averaging $T$ [3]. In this experiment, we set $N=512$ and $T=39$, and the A/D sampling frequency is $500 \mathrm{kHz} . M$ bins are averaged over $T$ times, and here, $M=66$. As a result, the frequency resolution is $0.98 \mathrm{kHz}$. Fig. 3 shows that the detection probability curve obtained from the USRP is approximately consistent with the theoretical curve obtained from [7].

\section{B. Communication part}

Circuits that generate the baseband and the IF signals are implemented in the FPGA board. In A-TX and B-TX, the

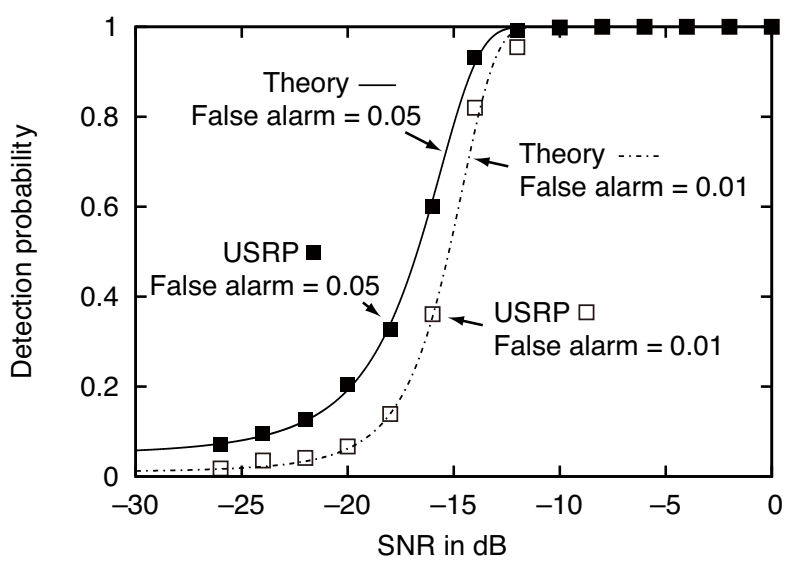

Fig. 3. Detection probability versus SNR.

TABLE I

MAJOR PARAMETERS OF COMMUNICATION PART

\begin{tabular}{l|r}
\hline Antenna gain of the directional antenna & $17 \mathrm{dBi}$ \\
Antenna gain of the omnidirectional antenna & $2.15 \mathrm{dBi}$ \\
Carrier frequency & $1.298 \mathrm{GHz}$ \\
TX power of A-TX (directional) & $2.0 \mathrm{dBm}$ \\
TX power of A-TX (omnidirectional) & $16.5 \mathrm{dBm}$ \\
TX power of B-TX (omnidirectional) & $8.6 \mathrm{dBm}$ \\
Antenna height of A-TX & $26 \mathrm{~m}$ \\
Antenna height of A-RX & $17.5 \mathrm{~m}$ \\
Antenna height of B-TX and B-RX & $1.5 \mathrm{~m}$ \\
FPGA & Rtratix EP1S25F780C5 \\
Intermediate frequency & $10.85 \mathrm{MHz}$ \\
Modulation & $\pi / 4$ shift QPSK \\
FIR filter & Root roll-off Nyquist \\
Symbol rate & $21.1914 \mathrm{kHz}$ \\
Packet length & $66 \mathrm{symbols}$ \\
Error detection & $\mathrm{CRC}-16$ \\
\hline
\end{tabular}

transceiver upconverts the IF signal generated by the FPGA board to the RF signal. In A-RX and B-RX, on the other hand, the transceiver downconverts the RF signal to the IF signal before demodulation by the FPGA board.

\section{EXPERIMENTAL SETUP AND RESULTS}

The major parameters of the communication part are shown in Table I. In this experiment, the WSND repeats the same process. First, the WSND receives $P_{\mathrm{A}, \mathrm{B}-\mathrm{TX}}$ from the $\mathrm{PC}$ through the USB. Here, $P_{\mathrm{A}, \mathrm{B}-\mathrm{TX}}$ is averaged over the last 39 cycles by the PC. On the basis of the averaged $P_{\mathrm{A}, \mathrm{B}-\mathrm{TX}}$, the WSND determines whether to transmit. An example of the sensing operation at B-TX is shown in Fig. 4.

The trial layout is shown in Fig. 5. In this experiment, the detection performance is evaluated at two positions, P1 and $\mathrm{P} 2$. A-RX uses a directional antenna (17-element loop Yagi antenna) and receives the weak signal from A-TX. Here, the transmission power of A-TX is $2.0 \mathrm{dBm}$. A $20-\mathrm{dB}$ attenuator is inserted into A-RX in order to eliminate the influence of the leakage power from A-TX, the shielding of which is not completely effective. For comparison, an omnidirectional antenna (half-wave whip antenna) is also employed in A-RX, and the transmission power of A-TX is set to $16.5 \mathrm{dBm}$. In both situations, the power levels received at A-RX, i.e., -89 $\mathrm{dBm}$, are the same. 


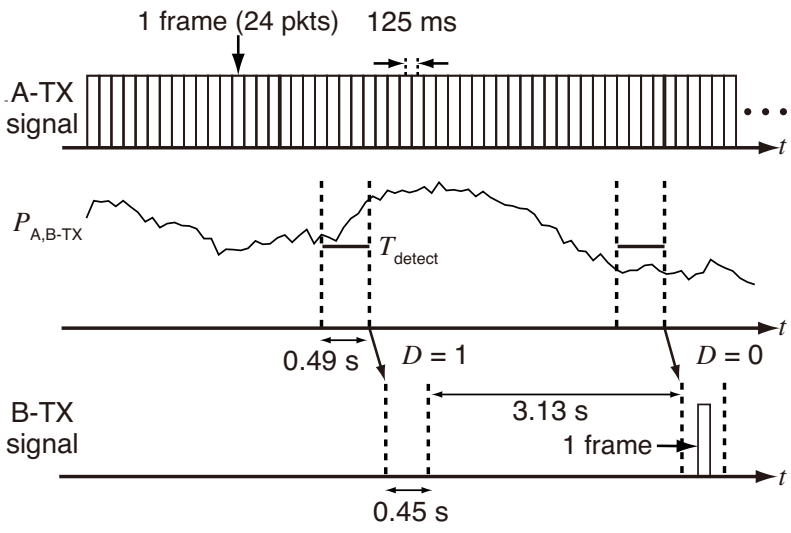

Fig. 4. Time structure of the experimental system.

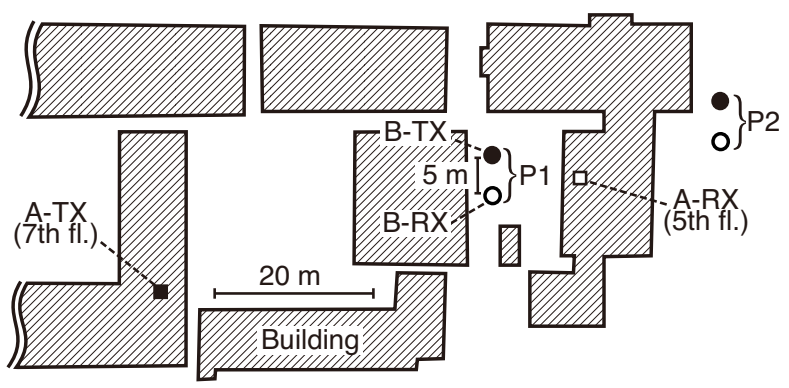

Fig. 5. Location of stations in the trial area.

Figs. 6 and 7 show the plot of the packet success ratio of A$\mathrm{RX}$ versus the transmission possibility of B-TX at P1 and P2. In each configuration, these values are obtained during a 3-min observation time. In this experiment, the number of received packets at B-RX is exactly equal to the number of transmitted packets at B-TX because there is no interference with BRX from A-TX. $T_{\text {detect }}$ is changed in 1-dB steps in order to compare the detection performance at different thresholds.

As shown in Fig. 6, an optimum threshold exists for the cognitive radio. When $T_{\text {detect }}=-110.8 \mathrm{dBm}, \mathrm{B}-\mathrm{TX}$ at $\mathrm{P} 2$ can transmit all packets while avoiding interference with A$\mathrm{RX}$, and B-TX at P1 can avoid interference with A-RX. Thus, if B-TX uses this threshold, it can opportunistically access the spectrum without causing interference with the high-priority A-RX.

On the other hand, as shown in Fig. 7, it is difficult for the cognitive radio to identify the optimum threshold for opportunistic secondary access without causing any interference with the high-sensitivity primary system. When $T_{\text {detect }}=$ $-113.8 \mathrm{dBm}, \mathrm{B}-\mathrm{TX}$ at $\mathrm{P} 2$ can transmit all packets without any interference with A-RX. However, B-TX at P1 causes harmful interference with A-RX. Moreover, when $T_{\text {detect }}=-115.8$ $\mathrm{dBm}, \mathrm{B}-\mathrm{TX}$ at P1 may not cause any interference with ATX, whereas B-TX at P2 largely prevents transmission. Thus, this lower detection threshold decreases the miss detection probability, while increasing the possibility of a false alarm under noise uncertainty.

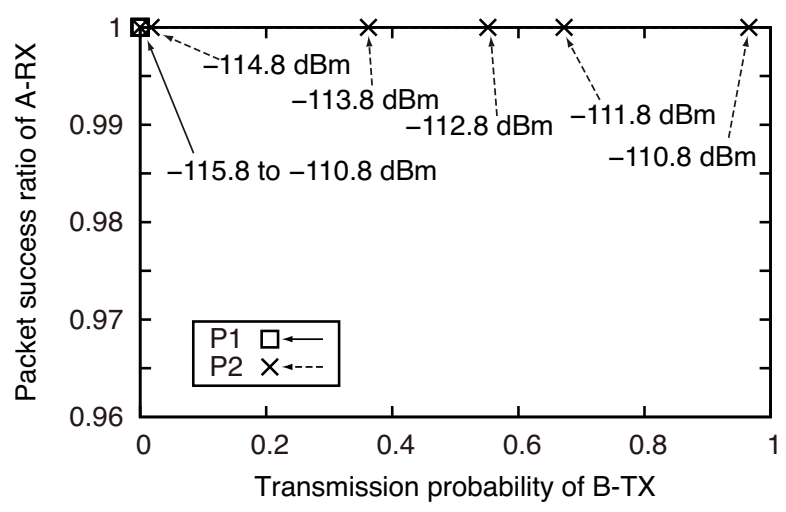

Fig. 6. Packet success ratio of A-RX using the omnidirectional antenna vs. transmission possibility of B-TX. Various detection thresholds are considered. The transmission power of A-TX is set to $16.5 \mathrm{dBm}$ in order to assume the scenario in which B-TX senses a strong signal from the nearby A-TX.

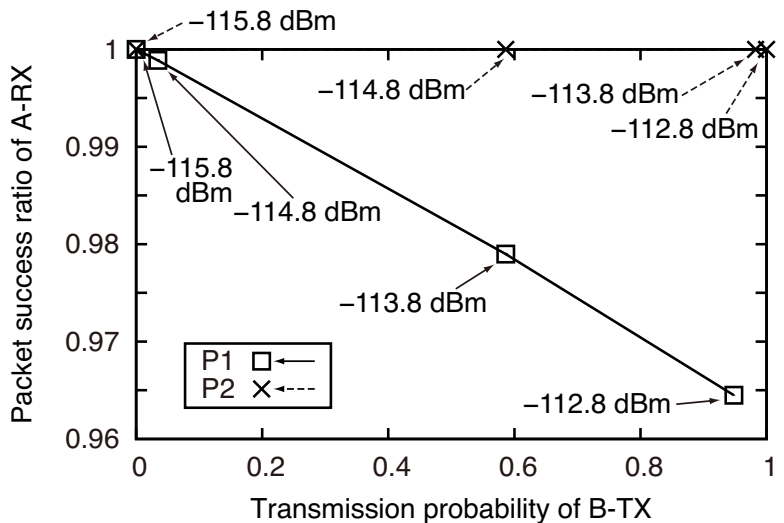

Fig. 7. Packet success ratio of A-RX using a directional antenna vs. transmission possibility of B-TX. Various detection thresholds are considered. The transmission power of A-TX is set to $2.0 \mathrm{dBm}$ in order to assume the scenario in which B-TX senses a weak signal from A-TX far away.

\section{CONCLUSION}

We have clarified the impact of the directional patterns of a primary system receiver in cognitive radio systems through an experimental case study. It is confirmed that the directional antenna of the primary system receiver may introduce additional tradeoff in selecting the threshold.

\section{REFERENCES}

[1] J. Mitola III and G. Q. Maguire Jr. , "Cognitive radio: Making software radios more personal," IEEE Personal Commun., vol. 6, no. 4, pp. 13-18, Aug. 1999.

[2] S. Haykin, "Cognitive radio: Brain-empowered wireless communications," IEEE J. Sel. Areas Commun., vol. 23, no. 2, pp. 201-220, Feb. 2005.

[3] D. Cabric, S. M. Mishra, A. Sahai and R. W. Brodersen, "Implementation issues in spectrum sensing for cognitive radios," Asilomar Conference on Signals, Systems, and Computers, vol. 1, pp. 772-776, Nov. 2004.

[4] S. Maharjan, K. Po and J. Takada, "Experimental study of energy detector prototype for cognitive radio system," IEICE Technical Report, SR200752, vol. 107, no. 352, pp. 43-48, Nov. 2007.

[5] P. J. Green and D. P. Taylor, "A real time cognitive radio test platform for public safety physical layer expriments," Proc. IEEE PIMRC'07, pp. 1-5, Sep. 2007.

[6] http://www.ettus.com/.

[7] H. Urkowitz, "Energy detection of unknown deterministic signals," Proc. of the IEEE, pp. 523-531, Apr. 1967. 